Draft version August 22, 2018

Typeset using $\mathrm{LAT}_{\mathrm{E}} \mathrm{X}$ preprint2 style in AASTeX61

\title{
INFERRING THE COMPOSITION OF DISINTEGRATING PLANET INTERIORS FROM DUST TAILS WITH FUTURE JAMES WEBB SPACE TELESCOPE OBSERVATIONS
}

\author{
Eva H. L. Bodman, ${ }^{1, *}{ }^{*}$ ason T. Wright,${ }^{2,3}$ Steven J. Desch, ${ }^{1}$ and Carey M. Lisse ${ }^{4}$
}

\author{
${ }^{1}$ School of Earth and Space Exploration, Arizona State University, P.O. Box 871404, Tempe, AZ 85287-1404, USA \\ ${ }^{2}$ Department of Astronomy $\mathcal{G}$ Astrophysics, The Pennsylvania State University, 525 Davey Laboratory, University \\ Park, PA, 16802, USA \\ ${ }^{3}$ Center for Exoplanets and Habitable Worlds, The Pennsylvania State University, 525 Davey Laboratory, University \\ Park, PA, 16802, USA \\ ${ }^{4}$ JHU-APL, 11100 Johns Hopkins Road, Laurel, MD 20723, USA
}

\begin{abstract}
Disintegrating planets allow for the unique opportunity to study the composition of the interiors of small, hot, rocky exoplanets because the interior is evaporating and that material is condensing into dust, which is being blown away and then transiting the star. Their transit signal is dominated by dusty effluents forming a comet-like tail trailing the host planet (or leading it, in the case of K2-22b), making these good candidates for transmission spectroscopy. To assess the ability of such observations to diagnose the dust composition, we simulate the transmission spectra from 5-14 $\mu \mathrm{m}$ for the planet tail assuming an optically-thin dust cloud comprising a single dust species with a constant column density scaled to yield a chosen visible transit depth. We find that silicate resonant features near $10 \mu \mathrm{m}$ can produce transit depths that are at least as large as those in the visible. For the average transit depth of $0.55 \%$ in the Kepler band for K2-22b, the features in the transmission spectra can be as large as 1\%, which is detectable with the JWST MIRI low-resolution spectrograph in a single transit. The detectability of compositional features is easier with an average grain size of $1 \mu \mathrm{m}$ despite features being more prominent with smaller grain sizes. We find most features are still detectable for transit depths of $\sim 0.3 \%$ in the visible range. If more disintegrating planets are found with future missions such as the space telescope TESS, follow-up observations with JWST can explore the range of planetary compositions.
\end{abstract}

Keywords: infrared: planetary systems — planets and satellites: composition — planets and satellites: interiors — planets and satellites: terrestrial planets planet-star interactions - techniques: spectroscopic

* NASA Postdoctoral Program Fellow, Nexus for Exoplanet System Science 


\section{INTRODUCTION}

During the Kepler mission (Borucki et al. 2009), a new subset of the ultra-short period planets ("USP planets," e.g. Sanchis-Ojeda et al. 2014), referred to as "disintegrating planets" was discovered. These appear to have large dusty tails as a result of their disintegration, presumably due to the intense irradiation of their host star (e.g. KIC 12557548, Rappaport et al. 2012). The transits of these disintegrating planets are characterized by a sharp and steep ingress followed by an extended tail-like egress or the reverse (extended ingress and steep egress) as in the case of K2-2b. Some systems also show a short brightening before the ingress and a brightening after the egress, suggestive of forward scattering in a dust tail. Currently, the most promising hypothesis for these systems is a low-mass (lunar or Mercury-like) disintegrating planet surrounded by escaping dusty effluents that form a comet-like tail much larger $(\sim 10 \mathrm{x})$ than the rocky planet itself (Rappaport et al. 2012). The dayside temperatures of these planets $(\sim 2000 \mathrm{~K})$ are high enough to evaporate rock and cause large mass loss. The transit depths of these planets are highly variable, possibly due to changes in the dust production rate which may linked to stellar activity (Croll et al. 2015; Kawahara et al. 2013).

Depending on the mechanism by which the material is leaving its parent body, the dust grains composing the comet-like tail may be a representative sample of the interior of the planet which can be examined through transmission spectroscopy. Practitioners of transmission spectroscopy of exoplanets typically must deal with a challenging geometry: the planetary transit itself is usually not difficult to detect, but the contribution to the signal from the exoplanetary atmosphere is often orders of magnitude smaller for small, rocky planets. Transmission spectroscopy has been widely applied to study the atmospheres of exoplanets with large scale heights, such as hot Jupiters, super-Neptunes, and super-Earths. These studies have significantly broadened our knowledge of planetary atmospheres. In the infrared (IR), molecules that are important to habitability, such as $\mathrm{H}_{2} \mathrm{O}, \mathrm{CH}_{4}, \mathrm{CO}_{2}$, and $\mathrm{CO}$, have been found in some planets using transmission spectroscopy (e.g., Barman 2008; Swain et al. 2008; Wakeford et al. 2013). Unlike most terrestrial exoplanets, the transit signal of disintegrating planets is almost entirely from the dust tail, making these planets strong candidates for transmission spectroscopy, like planets with atmospheres at large scale-heights.

Currently, three systems around main sequence stars have been discovered with apparent cometary tails of disintegrated material: KIC 12557548, KOI 2700, and K2-22. One white dwarf, WD 1145+017, also displays period transits of debris with cometary tails (Vanderburg et al. 2015), but the dust tail is likely due to tidal disruption unlike those in the other systems. There should be many more such objects in short-period orbits according to thermal evaporation models (Perez-Becker \& Chiang 2013), and current and future missions like K2 and TESS are expected to discover more of these objects around nearby bright stars that are well suited for characterization. DeVore et al. (2016) argue that there may be even more such planets detected via brightenings due to forward scattering by non-transiting dust.

\section{1. $K I C 12557548$}

KIC 12557548 was the first system discovered to have an orbiting disintegrating planet (Rappaport et al. 2012). The planet transits the mid$\mathrm{K}$ dwarf host star $\left(\mathrm{T}_{\text {eff }}=4300 \mathrm{~K}\right)$ at a short orbital period of $15.7 \mathrm{~h}$ and its transit light curve has the distinct asymmetric shape of a cometlike dusty tail. The transit duration is much longer than the crossing time of the planet $(\sim 0.1$ of the orbit), suggesting the tail is longer than even the size of the star. The deep transit depth 
$(<1.3 \%)$ and the comet-like tail were argued to be from plumes of dusty clouds condensed from the rock vapor from the planet's highly irradiated surface (2200 K) (Budaj 2013). The Kepler light curve of KIC $12557548 \mathrm{~b}$ also has a prominent pre-transit brightening and a weaker posttransit brightening, suggesting forward scattering by particles of radius of $0.1-1 \mu \mathrm{m}$ in the dusty tail (Budaj 2013). Schlawin et al. (2016) finds evidence for large particles in the tail from the flatness of the transmission spectrum, but this data was taken after the Kepler data during a smaller-than-average transit, which may indicate the system was in a weak period or the mechanism has changed.

The size of the dusty cloud, inferred from the transit depths, suggests a small rocky planet (based on its Hill radius) that has a rough mass loss rate of $1 \mathrm{M}_{\oplus} \mathrm{Gyr}^{-1}$ (Rappaport et al. 2012). Since it is extremely difficult on theoretical grounds to remove dust from large planets with substantial surface gravity, Perez-Becker \& Chiang (2013) concluded that KIC 12557548b is lower in mass than Mercury, and if the mass loss rate estimate is correct then the planet may be as small as 2 lunar masses, and may have lost $\sim 70 \%$ of its original mass, implying that it is in the final catastrophic disintegrating phase. This makes KIC 12557548b one of the smallest known exoplanets. (The smallest is Kepler 37b, Barclay et al. 2013.)

KIC 12557548's transit depth varies stochastically on timescales shorter than the orbital period, which might be caused by eruptions of dust (as in a cometary nucleus), variations in stellar radiation to the planet's surface, and/or volcanic activities at the planet's surface. All these hypotheses require more detailed theoretical and quantitative modeling to assess their credibility, and likely will imply different transmission properties of the cloud. There is evidence that its transit depth correlates with stellar rotation, likely due to the planet occulting
Table 1. System Properties

\begin{tabular}{lccc}
\hline \hline \multicolumn{1}{c}{ Property (Units) } & KIC 1255 & KOI 2700 & K2-22 \\
\hline Host Star Properties & & & \\
\hline Magnitude $J$ (mag) & 14.02 & 13.58 & 12.74 \\
Stellar Temperature (K) & 4500 & 4300 & 3830 \\
Surface gravity, log $g(\mathrm{cgs})$ & 4.62 & 4.71 & 4.65 \\
Metallicity [Fe/H] & -0.2 & -0.7 & 0.03 \\
\hline Planet Transit Properties & & & \\
\hline Orbital period (hr) & 15.68 & 21.84 & 9.146 \\
Transit length (hr) & 1.5 & $>5$ & 0.8 \\
Transit depth range (\%) & $0-1.4$ & $0.03-0.053$ & $0-1.3$ \\
Mean transit depth (\%) & 0.5 & 0.036 & 0.55 \\
\hline
\end{tabular}

References-Rappaport et al. (2012) for KIC 1255; Rappaport et al. (2014) for KOI 2700; Sanchis-Ojeda et al. (2015)

star spots rather than enhanced mass loss (Croll et al. 2015). The variability of the optical depth of the dusty cloud implies changes in the transit shape as well, but such variation is too small to detect for KIC $1255 \mathrm{~b}$ due to the faintness of its host $\operatorname{star}\left(K_{p}=15.7 \mathrm{mag}\right)$.

\subsection{KOI 2700}

KOI 2700b is reminiscent of KIC 12557548b in that its light curve shows a distinct asymmetric transit curve with a sharp ingress and a shallow egress, likely caused by a trailing dusty cometlike tail (Rappaport et al. 2014). Its average transit depth decreased by a factor of 2 over the course of the Kepler mission ( $\sim 4$ years), indicating a decrease of mass-loss rate or precession of the orbit out of the line of sight. The egress of the transit lasts for $\sim 25 \%$ of the orbital period $(21.84 \mathrm{hr})$, indicating a long lifetime for the dust grains and the tail. The transit depth of KOI $2700 \mathrm{~b}(\sim 360 \mathrm{ppm})$ is very shallow, suggesting a mass-loss rate an order of magnitude smaller than that of KIC $12557548 \mathrm{~b}$, and is too small to allow for analysis of transit-to-transit variation as with KIC 12557548b. The corresponding rocky core is expected to be as small as the Moon (Rappaport et al. 2014). The shal- 
low and decreasing transit depth of KOI 2700b ( $360 \mathrm{ppm})$ makes followup difficult; however, if the trend in depths reverses and the transits become deep again, it may make an excellent target.

\subsection{K2-22}

Another system that shows asymmetric transit light curves is the K2-22 system (SanchisOjeda et al. 2015), which includes a planet with a very short period of only 9.1457 hours around an M-dwarf host star $\left(\mathrm{T}_{\text {eff }} \sim 3800 \mathrm{~K}\right)$. Unlike KIC 12557548, the transit light curve of K2-22 does not have a significantly shallower egress. Instead, it shows a prominent posttransit brightening bump and a weaker pretransit brightening, both of which are better interpreted as forward scattering by a dominant leading dusty tail and a trailing tail with a large scale length of almost half of its host star's radius (Sanchis-Ojeda et al. 2015). Like KIC 12557548b, the transit depths are highly variable, ranging from $\leq 0.14 \%$ to $1.3 \%$. The shape of its transit light curves also appears to be variable. All these features point to dusty effluents escaped from the surface of a small rocky planet within a Moon-to-Mercury mass range.

Although the disintegrating planet hypothesis succeeds in explaining ground- and space-based observations, the particle sizes predicted by the two-dusty-tail model for K2-22b are not entirely self-consistent (Sanchis-Ojeda et al. 2015). In addition to grains being launched forwards along the orbit, the formation of a leading tail requires the ratio of radiation pressure forces to gravity to be low. This occurs for a lowluminosity host star for very small $(\lesssim 0.1 \mu \mathrm{m})$ or very large grains $(\gtrsim 1 \mu \mathrm{m})$. However, SanchisOjeda et al. (2015) found that the best fits of forward scattering bumps in the light curve prefer a grain size of $\sim 0.5 \mu \mathrm{m}$. The authors also found a weak color dependence in the transit depth for one of the dips they observed with ground-based spectroscopy which indicates dust with a grain size $\gtrsim 0.5 \mu \mathrm{m}$. Significant theoretical work is therefore still needed to reconcile all the observations and predictions. Since K2-22 is brighter than $\mathrm{KIC} 12557548\left(K_{p}=14.9\right)$, this star is currently the best target for follow-up observations, assuming the transit depths continue to exceed $1 \%$. A summary of these planetary system properties is listed in table 2 .

In this paper, we examine the detectability of the spectroscopic signatures of the composition of the dust grains in the disintegrating planet tails, in particular with regard to the capabilities of the upcoming James Webb Space Telescope (JWST) mission. In section 2, we discuss the composition of the dust that condenses out of vaporized rock material, assuming Earth-like composition. Then, in section 3 , we describe our simulated transmission spectra from different possible dust species that may be produced from the disintegration of interior planetary material. In section 3.1, we use our simulated spectra to estimate the detectability of compositional features with JWST.

\section{EXOPLANETARY MINERALOGY}

Disintegrating planets offer a tantalizing opportunity to measure exoplanetary interior composition directly. While the exact mechanism of producing the dust cloud is uncertain, the material is expected to be representative of an actual exoplanetary interior. The structure of the tail offers some compositional clues. Based on the lengths of tails and estimated dust grain lifetimes, and the sublimation properties of various minerals, van Lieshout et al. (2014) concluded that corundum $\left(\mathrm{Al}_{2} \mathrm{O}_{3}\right)$ and iron-rich silicates could be major components in the tail of KOI $2700 \mathrm{~b}$ and of the minerals considered, only corundum was consistent with KIC $12557548 \mathrm{~b}$ (van Lieshout et al. 2016). However, spectral information are more powerful. Since the dust cloud obscures the starlight at optical wavelengths, rudimentary characterization of composition can be done from the light curve tran- 
sits alone (van Lieshout et al. 2014, 2016). Supplementary IR spectra of disintegrating planet systems can constrain the dust compositions even more powerfully.

To assess the likely mineralogy of the dust, we adopt the hypothesis that the dust seen in tails that transit stars in disintegrating planet systems arises from the planets as follows. The planets are close enough to their host stars that their surface materials should be vaporizing. For the rocky materials that are expected to dominate, $\mathrm{Fe}, \mathrm{Mg}$-rich silicate minerals like olivine $\left((\mathrm{Mg}, \mathrm{Fe})_{2} \mathrm{SiO}_{4}\right)$ or pyroxene $\left((\mathrm{Mg}, \mathrm{Fe}) \mathrm{SiO}_{3}\right)$, this requires temperatures above about $1200 \mathrm{~K}$ at $P \sim 10^{-6}$ atm, to $1450 \mathrm{~K}$ for $P>10^{-3}$ atm (Ebel \& Grossman 2000; Ebel 2006). Ca,Al-rich silicates (e.g., hibonite, perovsite, grossite, melilite) and corundum $\left(\mathrm{Al}_{2} \mathrm{O}_{3}\right)$ vaporize at higher temperatures: $1450 \mathrm{~K}$ at $P \sim 10^{-6} \mathrm{~atm}$, to $2000 \mathrm{~K}$ at $P \sim 1 \mathrm{~atm}$ (Ebel \& Grossman 2000; Ebel 2006). No abundant minerals (based on cosmochemical/planetary abundances) are expected to survive at temperatures greater than about $2000 \mathrm{~K}$. A disintegrating planet with surface temperatures at or above $\sim 1200 \mathrm{~K}$ can build up an atmosphere of rock vapor that, if not gravitationally bound to the planet, will escape into space. As it escapes, the rock vapor expands and cools, allowing condensation of minerals from the vapor that evaporated. Whether the rock vapor condenses in the atmosphere or in the dust tail and the exact composition of the condensates is uncertain. Silicate clouds can form above magma oceans on Super-Earths assuming an adiabatic pressure profile (Schaefer \& Fegley 2009) but detailed modeling of disintegrating planet atmospheres and wind is needed.

In analogy to other condensates seen in other astrophysical systems, the grains that condense very likely will be micron-sized. Amoeboid olivine aggregates (AOAs) in chondritic meteorites consist of grains $5-20 \mu \mathrm{m}$ in diameter that condensed from the solar nebula (Scott \& Krot 2014). Models of dust condensation in asymptotic-giant-branch (AGB) outflows suggest grain growth to a maximum size of $\sim 1 \mu \mathrm{m}$ (Hoefner et al. 1996). The average radius of grains condensed from the winds of the red supergiant VY CMa is observationally constrained to be $\approx 0.5 \mu \mathrm{m}$ (Scicluna et al. 2015). Observations of dust condensed out of the Crab Nebula (core-collapse) supernova remnant likewise favor dust sizes just under $\sim 1 \mu \mathrm{m}$ in radius (Owen \& Barlow 2015). The maximum radii of dust grains in the interstellar medium are $0.25 \mu \mathrm{m}$ (Mathis et al. 1977). If our hypothesis that the dust from disintegrating planets is condensed from their rock vapor outflows, then the optical or, preferably, mid-infrared transmission spectra of these micron-sized dust grains can provide information on the stoichiometry of the vapor from which they condensed, and therefore the planets from which they are derived.

The minerals that would condense from these starting compositions would depend on the uncertain temperature to which the planet's surface is raised. If temperatures are not high enough to vaporize $\mathrm{Ca}$ or $\mathrm{Al}$, they would not be available to condense into minerals. Presence or absence of $\mathrm{Ca}$ or $\mathrm{Al}$ is more a function of temperature than intrinsic $\mathrm{Ca}$ or $\mathrm{Al}$ to $\mathrm{Si}$ ratios. However, some elements have similar condensation/vaporization temperatures and the ratio of those elements in condensed minerals would be diagnostic of the ratios in the evaporating surface material. In particular, the $50 \%$ condensation temperatures of $\mathrm{Fe}, \mathrm{Mg}$, and $\mathrm{Si}$ (in a solar-composition gas at $10^{-4} \mathrm{~atm}$ pressure) are $1328 \mathrm{~K}, 1327 \mathrm{~K}$, and $1302 \mathrm{~K}$, respectively; for comparison, those of $\mathrm{Ca}$ and $\mathrm{Al}$ are $1505 \mathrm{~K}$ and $1641 \mathrm{~K}$, respectively, and those of $\mathrm{Na}$ and $\mathrm{K}$ are $953 \mathrm{~K}$ and $1001 \mathrm{~K}$, respectively (Lodders 2003). Fe, $\mathrm{Mg}$, and $\mathrm{Si}$ are likely to vaporize together and condense together, with the ele- 
mental ratios in the dust grains reflecting the molar ratios in the current surface material.

In particular, it may be possible to distinguish whether dust is condensing from material derived from the crust, mantle (assuming the crust has been lost already), or core (assuming the mantle has been lost already) of a planet. For example, the Earth's continental crust is stoichiometrically majority silica (60wt\% $\mathrm{SiO}_{2}$ ). Crustal material also includes the minerals listed with wt\% in table 2 along with sub-percent fractions of other elements (Brown \& Mussett 1981). The Earth's oceanic crust is similar but distinct, being stoichiometrically less silica and more $\mathrm{FeO}, \mathrm{CaO}$, and $\mathrm{MgO}$ (Brown \& Mussett 1981). These crustal compositions contrast strongly with the composition of the Earth's mantle, which stoichiometrically has much more $\mathrm{MgO}$, more $\mathrm{FeO}$, and less $\mathrm{Al}_{2} \mathrm{O}_{3}$ (McDonough \& Sun 1995). A more detailed comparison of continental crust, oceanic crust and mantle composition is listed in table 2. In further contrast, the Earth's core is predominantly Fe metal $(\approx 86 \mathrm{wt} \%)$ and $\mathrm{Ni}$ metal $(\approx 4$ wt $\%)$, with light alloying elements of uncertain type and amounts, but arguably $\approx 6 \mathrm{wt} \%$ $\mathrm{Si}, \approx 3 \mathrm{wt} \% \mathrm{O}$, and $\approx 1 \mathrm{wt} \% \mathrm{~S}$ (Hirose et al. 2013).

The ratio of $\mathrm{Fe}$ and $\mathrm{Mg}$ atoms to $\mathrm{Si}$ atoms in the vapor determines whether the main condensates will be silica and pyroxenes [ $(\mathrm{Fe}+$ $\mathrm{Mg}) / \mathrm{Si}<1]$, pyroxenes and olivines $[1<$ $(\mathrm{Fe}+\mathrm{Mg}) / \mathrm{Si}<2]$, or olivines and periclase/wüstite $(\mathrm{MgO}$ and $\mathrm{FeO})[2<(\mathrm{Fe}+$ $\mathrm{Mg}) / \mathrm{Si}$ ]. These minerals are easily distinguished in the mid-infrared (Molster \& Kemper 2005; Wooden et al. 2007; Henning 2010), so IR observations should be able to constrain this elemental ratio. For an Earth-like composition, vaporized continental crust, with $(\mathrm{Fe}+\mathrm{Mg}) / \mathrm{Si} \approx$ 0.08 , should recondense overwhelmingly as silica, with some pyroxenes. Vaporized oceanic crust, with $(\mathrm{Fe}+\mathrm{Mg}) / \mathrm{Si} \approx 0.33$, should re-
Table 2. Earth Composition

\begin{tabular}{lccc}
\hline \hline Mineral & Cont. Crust & Ocean Crust & Mantle \\
\hline $\mathrm{SiO}_{2}$ & 60 & 49 & 45 \\
$\mathrm{Al}_{2} \mathrm{O}_{3}$ & 15 & 17 & 4 \\
$\mathrm{FeO}$ & 3.8 & 6.2 & 8 \\
$\mathrm{Fe}_{2} \mathrm{O}_{3}$ & 2.5 & 2.3 & \\
$\mathrm{CaO}$ & 5.5 & 12 & 3.5 \\
$\mathrm{MgO}$ & 3.1 & 6.8 & 38 \\
$\mathrm{Na} 2 \mathrm{O}$ & 3.0 & 2.6 & 0.3 \\
$\mathrm{~K}_{2} \mathrm{O}$ & 2.8 & 0.4 & 0.03 \\
$\mathrm{H}_{2} \mathrm{O}$ & 1.4 & 1.1 & \\
$\mathrm{CO}_{2}{ }^{a}$ & 1.2 & 1.4 & \\
$\mathrm{TiO}_{2}$ & 0.7 & 1.4 & 0.2 \\
\hline
\end{tabular}

References-Brown \& Mussett (1981) for crustal abundances; McDonough \& Sun (1995) for mantle abundances

$a_{\text {in carbonates }}$

condense predominantly as silica, but with a higher proportion of pyroxenes. Vaporized mantle, with $(\mathrm{Fe}+\mathrm{Mg}) / \mathrm{Si} \approx 1.42$, in contrast, should condense as a mixture of pyroxenes and olivines and not silica. Therefore the presence of silica may be diagnostic of crustal source material, while olivines may be diagnostic of mantle source material. Planet models are needed to test how diagnostic the $(\mathrm{Fe}+\mathrm{Mg}) / \mathrm{Si}$ ratio is for determining crust versus mantle material when the composition is significantly not Earth-like.

The $\mathrm{Fe} /(\mathrm{Mg}+\mathrm{Fe})$ ratio in silicates is easily observable through the shift in the $10 \mu \mathrm{m}$ silicate absorption feature it creates (e.g., Molster \& Kemper 2005; Wooden et al. 2007; Henning 2010), and the presence of Fe in silicates can also be inferred through the $0.9 \mu \mathrm{m}$ absorption feature (Cloutis \& Gaffey 1991). Silicates condensed from the Earth's continental crust, oceanic crust, and the mantle, would have molar ratios $\mathrm{Fe} /(\mathrm{Mg}+\mathrm{Fe}) \approx 0.52,0.37$, and 0.11 , respectively. Dust condensed from the outflow of an evaporating core would condense predominantly Fe metal, but to the ex- 
tent that silicates formed, they would lack $\mathrm{Mg}$ altogether. Given that an disintegrating exoplanet may have had a very different starting composition from the Earth, it may be difficult to unambiguously identify a molar ratio with continental or oceanic-like crust, but the differences in composition between crust, mantle, and core are stark and potentially identifiable. The exact ratio could provide information about the initial $\mathrm{Fe} / \mathrm{Mg}$ ratio in the planet or the redox state of the mantle.

These examples involving $\mathrm{Fe}, \mathrm{Mg}$, and $\mathrm{Si}$ demonstrate just some of the power of astromineralogical observations of disintegrating planet systems to derive information about planetary compositions. Much more information is potentially derivable through observations that identify Ca- or Al-bearing minerals, or metal grains, etc.

\section{DETECTABILITY WITH TRANSMISSION SPECTROSCOPY}

Both ground- and space-based resources will need to be brought to bear on the problem. Ground-based telescopes will be necessary for both their wavelength coverage (complementing the IR coverage of JWST) and for their monitoring capabilities. One major difficulty with observing disintegrating planets is, and will continue to be, their variable depths. Measurements of spectral slopes and features across different instruments must be made simultaneously, lest variations in a transit depth with time be mistaken for those with wavelength. Spaceborne telescope time is precious and difficult to schedule, which will make it difficult to measure deep transits on temperamental sources.

New ground-based and existing Kepler and K2 light measurements can yield statistics about the frequency of deep transits, since in many cases systems seem to go through active and quiescent phases. It may be possible to predict that at least one of, say, three consecutive
Table 3. Optical properties of minerals

\begin{tabular}{lcc}
\hline \multicolumn{1}{c}{ Mineral } & Wavelength Range & Ref \\
& $\mu \mathrm{m}$ & \\
\hline $\mathrm{Fe}$ (metallic iron) & $0.7-200$ & $\mathrm{O} 88$ \\
$\mathrm{C}$ (carbonaceous dust) & $.2-800$ & $\mathrm{D} 84$ \\
$\mathrm{SiO}_{2}$ (quartz) & $6-10000$ & $\mathrm{Z} 13$ \\
$\mathrm{MgSiO}_{3}$ (cryst. enstatite) & $2-98$ & $\mathrm{~J} 98$ \\
$\mathrm{Mg}_{2} \mathrm{SiO}_{4}$ (cryst. forsterite) & $0.2-2,2-8000$ & $\mathrm{~F} 01, \mathrm{Z} 11$ \\
$\mathrm{Fe}_{2} \mathrm{SiO}_{4}$ (cryst. fayalite) & $0.4-10,2-10000$ & $\mathrm{~F} 01$ \\
$\mathrm{Al}_{2} \mathrm{O}_{3}$ (corundum) & $0.5-400$ & $\mathrm{~K} 95$ \\
\hline
\end{tabular}

References-D84: Draine \& Lee (1984);F01: Fabian et al. (2001); J98: Jaeger et al. (1998); K95:Koike et al. (1995); O88: Ordal et al. (1988); Z11: Zeidler et al. (2011); Z13: Zeidler et al. (2013)

transits during an outburst will be deep enough to achieve one's science goals, with some confidence. If active phases persist long enough, monitoring by more easily-scheduled groundand space-based telescopes may allow for $J W S T$ to be triggered for targets of opportunity. Fortunately, the nature of USP planets is that over the course of a small number of days a few or several transits can be observed, increasing the likelihood that a sufficiently deep transit will be observed if conditions are favorable. Since the transit depth reaches over $1 \%$ and it is the brightest of the three, K2-22 is currently the best target for follow-up. The planet's 9 hour orbit also allows for quick establishment of its ephemeris and transit depth statistics.

Here, we consider seven dust species (listed in table 3): $\mathrm{Fe}$ and $\mathrm{C}$, two species that primarily contribute to the spectral continuum; quartz $\left(\mathrm{SiO}_{2}\right)$; enstatite $\left(\mathrm{MgSiO}_{3}\right)$, forsterite $\left(\mathrm{Mg}_{2} \mathrm{SiO}_{4}\right)$, and fayalite $\left(\mathrm{Fe}_{2} \mathrm{SiO}_{4}\right)$, three silicates; and corundum $\left(\mathrm{Al}_{2} \mathrm{O}_{3}\right)$, a likely marker for crustal material. Only forsterite and fayalite are included from the olivine mineral family because those two minerals are the end-members, containing only $\mathrm{Mg}$ or Fe. Typical olivine is a mixture of these two minerals and displays a 
blend of the spectral features from those two minerals. The tail will more likely contain a mixture of both forsterite and fayalite. We did not include any iron-rich pyroxenes, as condensation of enstatite over ferrosilite $\left(\mathrm{FeSiO}_{3}\right)$ is highly favored, and Fe is overwhelmingly seen in solar system silicates as fayalite.

A spherical grain with radius $r$ has an extinction cross section $\sigma(\lambda, r)=\pi r^{2} Q_{\text {ext }}$, where $Q_{\text {ext }}(\lambda, r)$ in the monochromatic extinction efficiency. We calculate the extinction efficiencies using Mie theory and the references for the index of refraction data for each dust species is listed in table 3 . The index of refraction was extrapolated to shorter wavelengths as needed, see the wavelength range of the data in table 3 . We assume a simple power-law grain size distribution, $d n=r^{-a} d r$ with an effective grain radius of $r_{\text {eff }}=\int r^{1-a} d r / \int r^{-a} d r$. We average over the grain size distribution to calculate an effective monochromatic extinction cross section,

$$
\bar{\sigma}(\lambda)_{\mathrm{eff}}=\frac{\pi \int_{0.01}^{r_{\max }} Q_{\mathrm{ext}}(\lambda, r) r^{2-a} d r}{\int_{0.01}^{r_{\max }} r^{-a} d r}
$$

Since the grain size in the planet tails has been constrained from forward scattering and wavelength dependency of the transit depths to 0.1

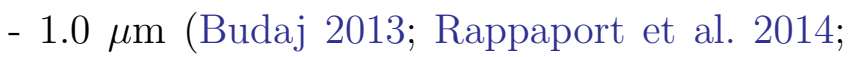
Sanchis-Ojeda et al. 2015), we consider two size distributions to approximate the observed upper and lower limits of the effective dust grain size. For the small grain size limit, we set the maximum radius $\left(r_{\max }\right)$ at $2.0 \mu \mathrm{m}$ and set the exponent for each species such that $r_{\text {eff }}=0.1 \mu \mathrm{m}$; for the large grain size limit, $r_{\max }=5.0 \mu \mathrm{m}$ and $r_{\text {eff }}=1.0 \mu \mathrm{m}$.

The effective total extinction cross sections for each size distribution are shown in Fig. 1 along with the effective scattering and absorption cross sections which are related by $\bar{\sigma}_{\text {ext }}=\bar{\sigma}_{\text {sca }}+\bar{\sigma}_{\text {abs }}$. For wavelengths shorter than $\sim 6 \mu \mathrm{m}$, the extinction cross section is dominated by scattering, resulting in a featureless curve that depends on the grain size with a degeneracy with composition and cannot be distinguished in this wavelength range alone. In the 3-7 $\mu \mathrm{m}$ region, the extinction cross section rapidly decreases with the scattering efficiency for all compositions except iron and carbon, which remain approximately flat to $\sim 15$ $\mu \mathrm{m}$. Longward of $\sim 7 \mu \mathrm{m}$, silicates have distinguishing spectral features, as does corundum at $\sim 10 \mu \mathrm{m}$. Since the unique compositional features occur in the mid-IR, we focus the rest of our analysis over the wavelength range for the JWST MIRI spectrograph $(\sim 5-14 \mu \mathrm{m})$.

To simulate planet dust tail transits, we assume an optically-thin dust cloud with a constant opacity occulting the star. This is an unrealistic simplification; the actual dust tails will be expected to have decreasing opacity with increasing distance from the planet, as well as a decreasing grain size with increasing distance. However, the deepest part of the transit occurs when the dense region of the dust tail that is slightly leading the planet is occulting, so the effects from the changes in opacity and grain size should be small. We also neglect limb darkening and assume the cloud is small compared to the star. The wavelength-dependent transit depth is

$$
\delta(\lambda)=\frac{N \bar{\sigma}(\lambda)_{\mathrm{eff}}}{\pi R_{\star}^{2}}
$$

where $N$ is the number of dust grains in the cloud and $\pi R_{\star}^{2}$ is the stellar surface. We scale $N$ to the transit depth in the visible.

\subsection{Results}

We calculate spectra using K2-22 as an example, scaling $N$ so that the transit depth is the average transit depth for K2-22 (0.55\%) in the Kepler band. To estimate the errors, we use the JWST transit error simulation code PANDExo (Batalha et al. 2017) with the K2-22 system properties listed in table 2 and the JWST MIRI Low Resolution Spectrograph (LRS) on slitless mode. We assume a conservative noise 

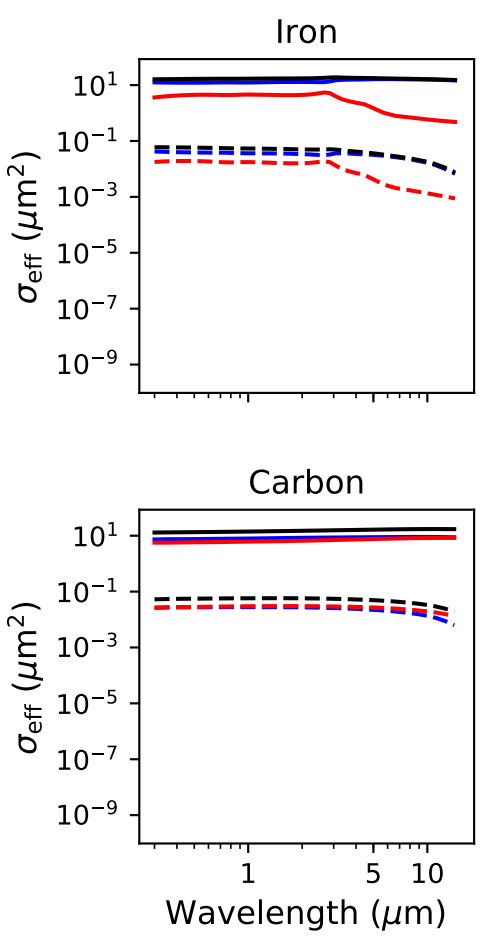
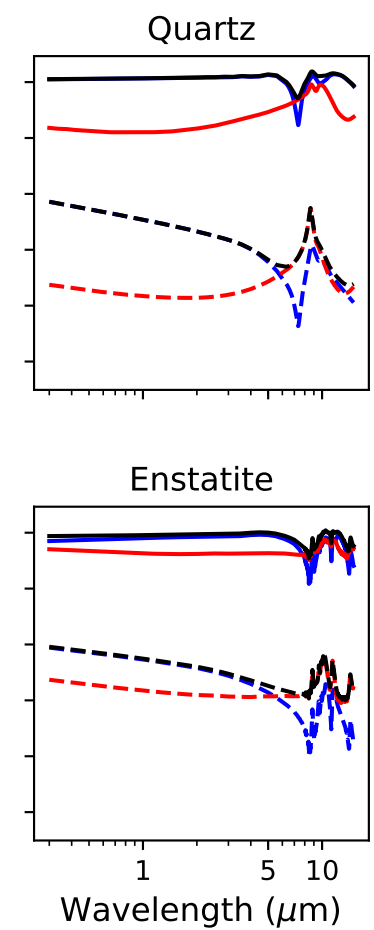

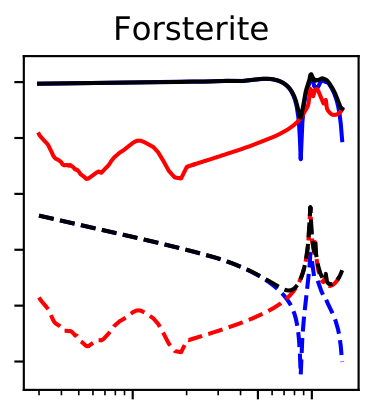

Fayalite

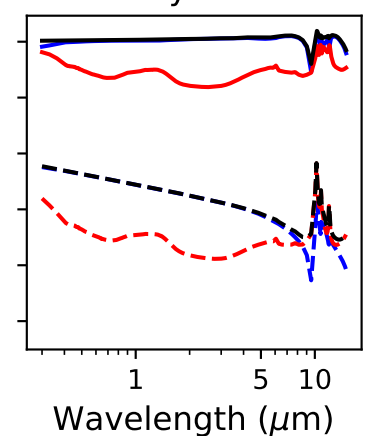

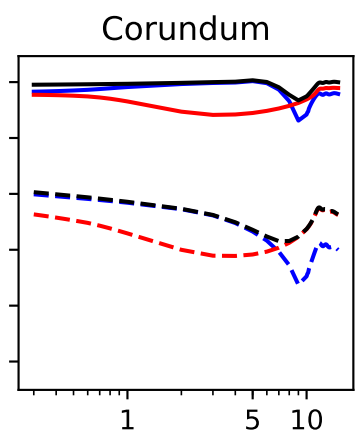

Wavelength $(\mu \mathrm{m})$

Figure 1. Extinction, scattering, and absorption cross sections for each of the dust species in black, blue and red respectively. The solid lines display the cross sections for the large grain size distribution and the dashed lines show the small grain size distribution. Scattering dominates at the shorter wavelengths, resulting in a smooth power law dependence that has a size and composition degeneracy that is particularly problematic for the larger grain sizes. Unique compositional features appear at wavelengths longward of $\sim 7 \mu \mathrm{m}$ for the silicates and corundum.

floor of $50 \mathrm{ppm}$ and calculate the results for a single transit with equal in-transit and outof-transit integration times. We calculate the transmission spectra for a cloud of a single composition, shown in Fig. 2 for large grain size $\left(r_{\text {eff }}=1.0 \mu \mathrm{m}\right)$ and small grain size distributions $(0.1 \mu \mathrm{m})$ in the upper and lower plots, respectively. The left plots show the unbinned data at a resolution of $R=100$ with every 35 th error bar (reduced for clarity) and the right plots display the data binned to $R=10$.

The carbon and iron dust grains produce featureless spectra that are distinguished only by their relative depths at shorter wavelengths. Corundum has a single wide peak beginning near $12 \mu \mathrm{m}$ with a small transit depth between 8 and $10 \mu \mathrm{m}$ that distinguishes it from the other dust species. Quartz has a peak near $8.5 \mu \mathrm{m}$ which is distinguishable for both grain size distributions. For the silicates, fayalite and forsterite have peaks at near $10 \mu \mathrm{m}$ that are far enough apart to be distinguished even in a low-resolution spectrum. Enstatite and fayalite both have peaks near $10.5 \mu \mathrm{m}$, making them indistinguishable from this feature alone, but have distinct absorption properties between 6 and $8 \mu \mathrm{m}$ for larger grains. However, for smaller grains, this degeneracy would only be distinguishable for resolutions of $R>10$. For each of the species with peaks, the depth is similar to that in the visible to about double the depth, although the size of the features is sensitive to the grain size.

Because these features are quite broad, lowresolution $(R \approx 10)$ spectra are sufficient to distinguish them, which allows us to bin the MIRI spectra enough to achieve sufficient SNR to detect them in the case of large grain size distri- 

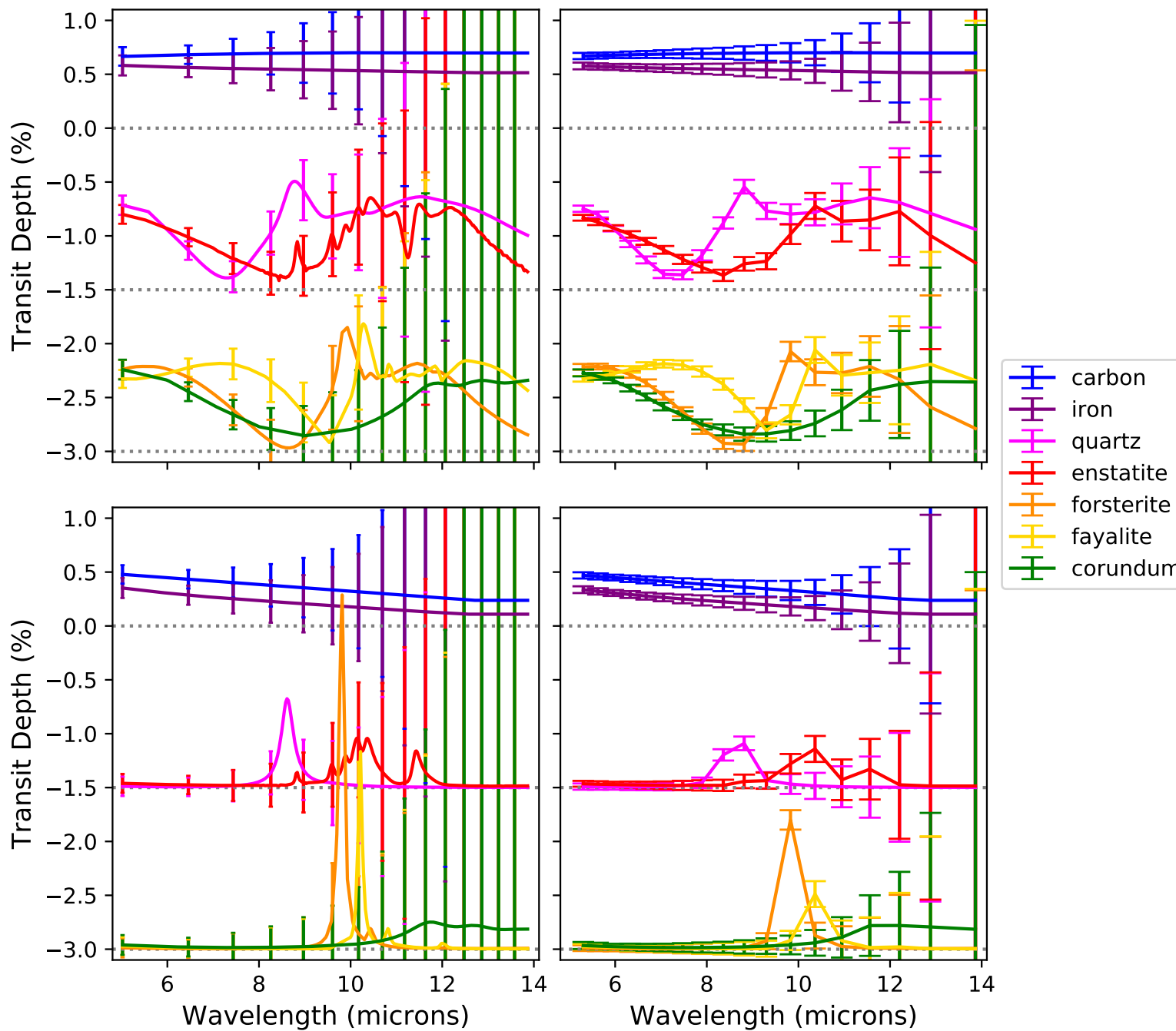

Figure 2. Model spectra of a single transit assuming the average transit depth for K2-22b (0.55\%) in the visible for expected dust species (listed in Table 3) with the two size distributions. The large grain sizes $\left(r_{\text {eff }}=1.0 \mu \mathrm{m}\right)$ are in the upper plots and the small grain sizes $\left(r_{\text {eff }}=0.1 \mu \mathrm{m}\right)$ are in the lower plots. The left plots are the unbinned spectra with only every 35th error bar shown and the right plots are binned to $\mathrm{R}=10$. The plots do not include random variance so that the spectral features are more easily seen and the depths are offset for clarity with dotted line marking 0 transit depths for that offset. Colors mark the different dust species: blue for iron, purple for carbon, pink for quartz $\left(\mathrm{SiO}_{2}\right)$, red for enstatite $\left(\mathrm{MgSiO}_{3}\right)$, orange for forsterite $\left(\mathrm{Mg}_{2} \mathrm{SiO}_{4}\right)$, yellow for fayalite $\left(\mathrm{Fe}_{2} \mathrm{SiO}_{4}\right)$, and green for corundum $\left(\mathrm{Al}_{2} \mathrm{O}_{3}\right)$. Spectral features longward of $9 \mu \mathrm{m}$ are only detectable in the binned spectra.

bution. For the high-resolution $(\mathrm{R}=100)$ unbinned data, the error size is comparable to the transit depth longward of $9 \mu \mathrm{m}$ so only the quartz feature is detectable. The large grains broaden spectral features and create a small amount of background extinction that is weakly wavelength-dependent, decreasing with increas- ing wavelength particularly between $6-8 \mu \mathrm{m}$. Both of these effects in general create deeper spectral features that are easier to detect. Small grains have deeper spectral features but also thinner features which reduce in size quickly when the data are binned. A higher-resolution binning would be preferable if the grains are 

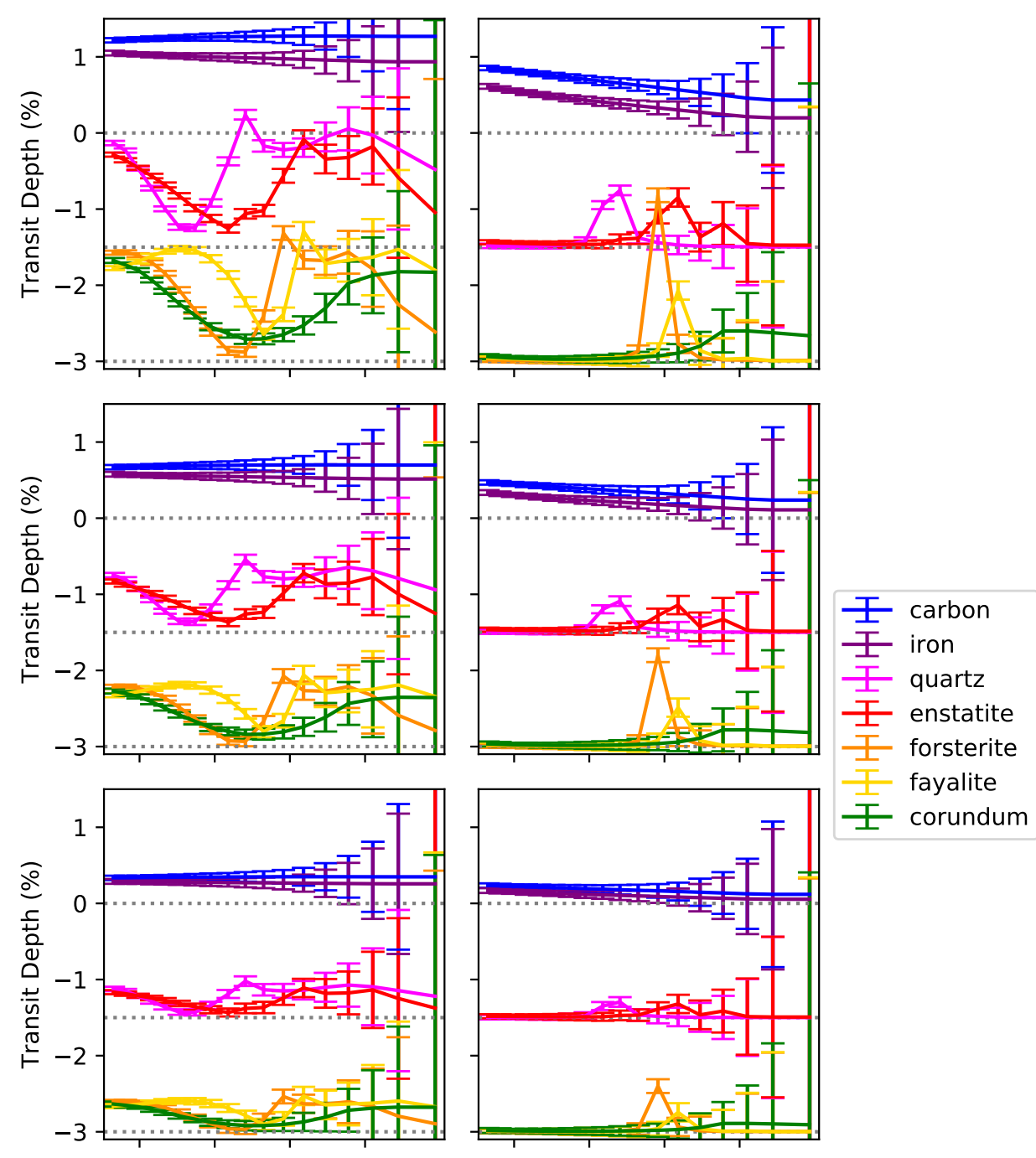

fayalite

王 corundum
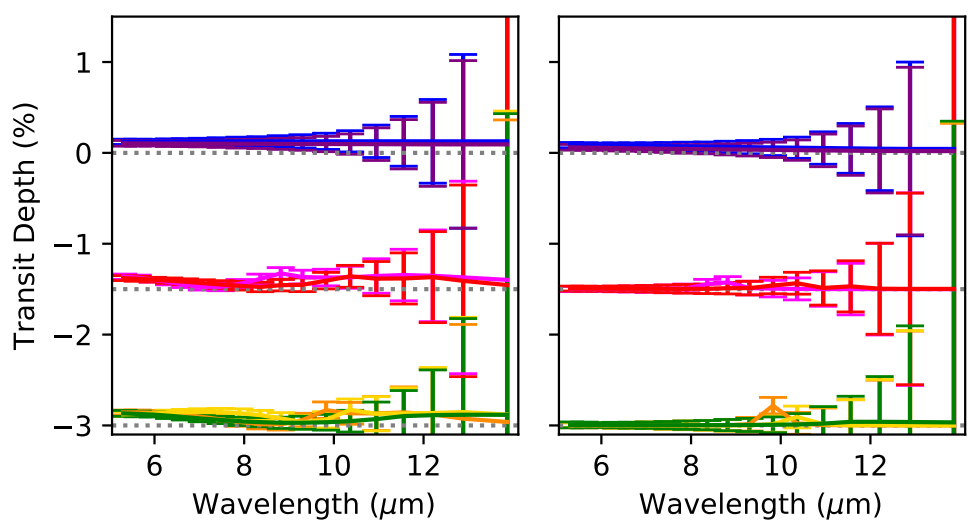

Figure 3. Low-resolution $(\mathrm{R}=10)$ model spectra of a single transit for expected dust species for $1 \%, 0.55 \%$, $0.275 \%$, and $0.1 \%$ dips in the Kepler bandpass, plotted in order from top to bottom. The left plots are for the large grain size distribution $\left(r_{\text {eff }}=1.0 \mu \mathrm{m}\right)$ and the right plots are small grains $\left(r_{\text {eff }}=0.1 \mu \mathrm{m}\right)$. The same colors as in Fig. 2 mark the dust species and are offset in the same manner. Some features remain detectable down to a $0.275 \%$ transit. The $13 \mu \mathrm{m}$ corundum features remains undetectable for small grains at a $1 \%$ transit depth and marginally detectable for large grains. 
small since it would make the features more prominent. The enstatite and fayalite features only have a marginally-detectable feature for the small grain size distribution while quartz and forsterite would have strong detections. For corundum, the $13 \mu \mathrm{m}$ feature would only be a weak (1-2 $\sigma$ ) detection for either grain size, but if the grains are large, the relative absorption between 6 and $8 \mu \mathrm{m}$ and the lack of a feature at $10 \mu \mathrm{m}$ make corundum distinguishable.

In Fig. 3, we examine a range of transit depths observed by Kepler for this system. As expected, the features are larger with a larger transit, but even at $1 \%$ the corundum feature is only marginally significant for small grains. At this larger transit depth, the breadth of the enstatite feature at $10.5 \mu \mathrm{m}$ compared to fayalite becomes marginally detectable. For half the average depth $(0.275 \%)$, the silicate features are still detectable for large grains but only forsterite is detectable for small grains. At $0.1 \%$, no features for either grain size distribution have detectable compositional signatures. The slopes of the spectra between 6-8 $\mu \mathrm{m}$ are still significantly different for some of the species, but the slope is degenerate with size in this wavelength region as the extinction cross-section is still dominated by the scattering cross-section. Only weak compositional constraints could be made at this small transit depth. For a realistic cloud with multiple dust species, these features will be blended so the minimum visible transit depth required for detection of silicates is at least $0.3 \%$.

\section{CONCLUSIONS}

We have calculated the detectability by infrared observations, in particular using JWST, of different mineral condensates in the tails of disintegrating planets, and encourage such observations. The mineralogy of the dust will give us the first direct measurements of the chemical composition of the interiors of exoplanets and this can be be done with the capabilities of
JWST. Low-resolution transmission spectra allow for sufficiently high SNR in a single transit for a strong detection of any ferromagnesium silicate features that may exist in the dust tails of these planets if the transit depth in the Kepler bandpass is at least $0.3 \%$. Since the average depth for $\mathrm{K} 2-22 \mathrm{~b}$ is $0.55 \%$, we should be able to observe at least one deep enough transit in a relatively low number of observations. With concurrent observations in the visible, several spectra may be combined to improve SNR despite the stochastic nature of the transit depths. If KIC 12557548b becomes more active again, then that system could also be characterized by JWST, but KOI 2700b transit depths are currently too small.

If the dust is condensing from rocky vapor from a molten surface, the relative abundances in the dust species will allow us to determine whether it is crustal, mantle, or core material that is escaping. We expect the crust would produce more Al-bearing minerals than interior material, while the core would produce primarily iron-bearing minerals. Since the crustal layer is thin, crustal material would disintegrate in a short period of time compared to the mantle. Detection of crustal material may be less likely, but if discovered it would constrain the time for which the planet has been disintegrating. If the dusty affluents are from volcanic plumes, then the presence of volatile elements such as carbon may be an indicator.

Assuming mantle material is escaping and the dust is representative of the mantle composition, determining the abundances of the $\mathrm{Mg}$ , Si-, and Fe-bearing minerals can be used to derive the $\mathrm{Mg} / \mathrm{Si}$ and $\mathrm{Fe} / \mathrm{Si}$ ratios of the dust which then can constrain these ratios in the planet's interior. Core size probably correlates directly with $\mathrm{Fe} / \mathrm{Si}$, and many important geophysical properties of the mantle - water storage capacity, thermal conductivity, rheology, etc. - depend on the mineralogy of the man- 
tle. If more disintegrating planets are found in future missions such as TESS, we can use these planets to test how good of a proxy the stellar composition is for the composition of the planet, although precise stellar composition observations are also required to test this.

The authors thank Brad Foley for useful discussions, Daniel Jontof-Hutter for comments, and Natasha Batalha for assistance with the Pandexo code.
This work was partially supported by funding from the Center for Exoplanets and Habitable Worlds, which is supported by the Pennsylvania State University, the Eberly College of Science, and the Pennsylvania Space Grant Consortium. E.H.L.B.'s research was supported by an appointment to the NASA Postdoctoral Program with the Nexus for Exoplanet System Science, administered by Universities Space Research Association under contract with NASA.

\section{REFERENCES}

Barclay, T., Rowe, J. F., Lissauer, J. J., et al. 2013, Nature, 494, 452

Barman, T. S. 2008, ApJL, 676, L61

Batalha, N. E., Mandell, A., Pontoppidan, K., et al. 2017, PASP, 129, 064501

Borucki, W., Koch, D., Batalha, N., et al. 2009, in IAU Symposium, Vol. 253, IAU Symposium, 289-299

Brown, G. C., \& Mussett, A. E. 1981, The inaccessible earth (London: Allen \& Unwin)

Budaj, J. 2013, A\&A, 557, A72

Cloutis, E. A., \& Gaffey, M. J. 1991, J. Geophys. Res., 96, 22

Croll, B., Rappaport, S., \& Levine, A. M. 2015, MNRAS, 449, 1408

DeVore, J., Rappaport, S., Sanchis-Ojeda, R., Hoffman, K., \& Rowe, J. 2016, MNRAS, 461, 2453

Draine, B. T., \& Lee, H. M. 1984, ApJ, 285, 89

Ebel, D. S. 2006, Condensation of Rocky Material in Astrophysical Environments, ed. D. S. Lauretta \& H. Y. McSween, 253-277

Ebel, D. S., \& Grossman, L. 2000, GeoCoA, 64, 339

Fabian, D., Henning, T., Jäger, C., et al. 2001, A\&A, 378, 228

Henning, T. 2010, ARA\&A, 48, 21

Hirose, K., Labrosse, S., \& Hernlund, J. 2013, Annual Review of Earth and Planetary Sciences, 41, 657

Hoefner, S., Fleischer, A. J., Gauger, A., et al. 1996, A\&A, 314, 204

Jaeger, C., Molster, F. J., Dorschner, J., et al. 1998, A\&A, 339, 904
Kawahara, H., Hirano, T., Kurosaki, K., Ito, Y., \& Ikoma, M. 2013, ApJL, 776, L6

Koike, C., Kaito, C., Yamamoto, T., et al. 1995, Icarus, 114, 203

Lodders, K. 2003, ApJ, 591, 1220

Mathis, J. S., Rumpl, W., \& Nordsieck, K. H. 1977, ApJ, 217, 425

McDonough, W. F., \& Sun, S.-s. 1995, Chemical Geology, 120, 223

Molster, F., \& Kemper, C. 2005, SSRv, 119, 3

Ordal, M. A., Bell, R. J., Alexander, Jr., R. W., Newquist, L. A., \& Querry, M. R. 1988, ApOpt, 27, 1203

Owen, P. J., \& Barlow, M. J. 2015, ApJ, 801, 141

Perez-Becker, D., \& Chiang, E. 2013, MNRAS, 433, 2294

Rappaport, S., Barclay, T., DeVore, J., et al. 2014, ApJ, 784, 40

Rappaport, S., Levine, A., Chiang, E., et al. 2012, ApJ, 752, 1

Sanchis-Ojeda, R., Rappaport, S., Winn, J. N., et al. 2014, ApJ, 787, 47

Sanchis-Ojeda, R., Rappaport, S., Pallè, E., et al. 2015, ApJ, 812, 112

Schaefer, L., \& Fegley, B. 2009, ApJL, 703, L113

Schlawin, E., Herter, T., Zhao, M., Teske, J. K., \& Chen, H. 2016, ApJ, 826, 156

Scicluna, P., Siebenmorgen, R., Wesson, R., et al. 2015, A\&A, 584, L10

Scott, E. R. D., \& Krot, A. N. 2014, Chondrites and Their Components, ed. A. M. Davis, 65-137

Swain, M. R., Vasisht, G., Tinetti, G., et al. 2008, ArXiv e-prints, arXiv:0812.1844 
van Lieshout, R., Min, M., \& Dominik, C. 2014, A\&A, 572, A76

van Lieshout, R., Min, M., Dominik, C., et al. 2016, A\&A, 596, A32

Vanderburg, A., Johnson, J. A., Rappaport, S., et al. 2015, Nature, 526, 546

Wakeford, H. R., Sing, D. K., Deming, D., et al. 2013, MNRAS, 435, 3481
Wooden, D., Desch, S., Harker, D., Gail, H.-P., \& Keller, L. 2007, Protostars and Planets V, 815 Zeidler, S., Posch, T., \& Mutschke, H. 2013, A\&A, 553, A 81

Zeidler, S., Posch, T., Mutschke, H., Richter, H., \& Wehrhan, O. 2011, A\&A, 526, A68 